\title{
Treatment of uncertainty in conservation under climate change
}

\section{Kujala, Heini}

2013

Kujala , H , Burgman , M A \& Moilanen , A 2013 , ' Treatment of uncertainty in conservation under climate change ' , Conservation Letters , vol. 6 , pp. 73-85 . https://doi.org/10.1111/j.1755-263X.2012.00299.x

http://hdl.handle.net/10138/43063

https://doi.org/10.1111/j.1755-263X.2012.00299.x

publishedVersion

Downloaded from Helda, University of Helsinki institutional repository.

This is an electronic reprint of the original article.

This reprint may differ from the original in pagination and typographic detail.

Please cite the original version. 


\title{
Treatment of uncertainty in conservation under climate change
}

\author{
Heini Kujala ${ }^{1}$, Mark A. Burgman ${ }^{2}$, \& Atte Moilanen ${ }^{1}$ \\ ${ }^{1}$ Department of Biosciences, University of Helsinki, P.O. Box 65 (Viikinkaari 1), Fl-00014, Helsinki, Finland \\ ${ }^{2}$ School of Botany, University of Melbourne, Parkville, Victoria 3010, Australia
}

\author{
Keywords \\ Biodiversity; conservation planning; epistemic \\ uncertainty; global warming; human decision \\ uncertainty; linguistic uncertainty; uncertainty \\ analysis.

\section{Correspondence} \\ Heini Kujala, Department of Biosciences, \\ University of Helsinki, P.O. Box 6500014, \\ Helsinki, Finland. Tel: +358 9191 57922; \\ fax: +358919157694 \\ E-mail: heini.kujala@helsinki.fi, \\ heini.kujala@unimelb.edu.au

\section{Received} \\ 14 March 2012 \\ Accepted \\ 13 September 2012 \\ Editor \\ Hugh Possingham \\ doi: 10.1111/j.1755-263X.2012.00299.x
}

\begin{abstract}
Climate change is an important threat to biodiversity globally, but there are major uncertainties associated with its magnitude and ecological consequences. Here, we investigate how three major classes of uncertainty, linguistic uncertainty, epistemic uncertainty (uncertainty about facts), and human decision uncertainty, have been accounted for in scientific literature about climate change. Some sources of uncertainty are poorly characterized and epistemic uncertainty is much more commonly treated than linguistic or human decision uncertainty. Furthermore, we show that linguistic and human decision uncertainties are relatively better treated in the literature on sociopolitics or economics than in natural sciences, which often overlook communication between stakeholders and socioeconomic consequences. As uncertainty can significantly influence implementation of conservation, we discuss uncertainties associated with some commonly proposed conservation adaptation actions to mitigate climate change. There may be major differences between strategies, with implications on how they should be viewed in conservation planning. We conclude that evaluating conservation strategies in terms of different types of uncertainty will facilitate communication between disciplines and stakeholders. While accounting for uncertainties in a quantitative manner is difficult and data demanding, even qualitative appreciation about the uncertainties inherent in conservation strategies can facilitate and improve decision making.
\end{abstract}

\section{Introduction}

Global biodiversity conservation faces serious challenge, as species extinction rates elevated by habitat loss and other drivers are expected to further increase with climate change (Sala et al. 2000; Travis 2003; Opdam $\&$ Wascher 2004; Bellard et al. 2012). The ultimate consequences remain unknown because of the uncertainty and complexity of the climate system and its socioecological feedbacks, and the rapid pace of change, which may create novel climates without historical analogues (Dawson et al. 2011; Polasky et al. 2011). Consequently, global conservation efforts struggle with uncertainties that hamper effective decision making (Ludwig et al.

Re-use of this article is permitted in accordance with the Terms and Conditions set out at http://wileyonlinelibrary.com/ onlineopen\#OnlineOpen_Terms
2001; Ascough II et al. 2008). Broadly, uncertainty about climate change follows from uncertainty about (1) biophysical processes, (2) human actions that impact climate change, (3) ecological consequences of climate change, (4) feedbacks between processes, and (5) the objectives of human decision makers and other participants.

Uncertainty, in itself, is not a simple, well-defined concept that is easy to account for in research and management. Different types of uncertainties have different implications for research or operational decision making (Ludwig et al. 2001). In this work, we classify uncertainty in three major categories: (1) linguistic uncertainty, (2) human decision uncertainty, which is uncertainty about the world views, objectives, and preferences of stakeholders, and (3) epistemic uncertainty, which is uncertainty about facts. Because conservation decision making spans across multiple disciplines, we investigate how these 
uncertainties have been treated in climate change studies of different research fields to evaluate how different disciplines address uncertainty. We also evaluate types of uncertainty associated with commonly proposed conservation strategies. The broad aim of this work is to structure our understanding about types of uncertainty, and to illustrate how uncertainties can be evaluated for conservation strategies proposed for climate change adaptation.

\section{Methods \\ Types of uncertainty}

Uncertainty is incomplete knowledge or lack of certainty about a particular subject (Knight 1921). There are several classifications of uncertainty, and while many sources of uncertainty have been recognized, there remains some disagreement about the kinds of uncertainty, their characteristics and relative importance (Ascough II et al. 2008). Table 1 shows the classification of uncertainties we employ in the present study. We use a high-level classification of linguistic, epistemic and human decision uncertainty, and within each class we identify subclasses of uncertainty.

The majority of uncertainty investigations have concentrated on epistemic (knowledge based) uncertainty, which can be attributed to either a lack of knowledge about biogeophysical processes, or, to natural variability, which is associated with seemingly random variation in natural or anthropogenic processes-things that are beyond our measuring capabilities (Deser et al. 2012). From a conservation perspective, accurately characterizing and reducing epistemic uncertainty is crucial for effective conservation resource allocation. For instance, apparent inconsistencies between scientific studies that arise from epistemic uncertainty may result in policy makers, stakeholders, and the general public questioning the overall usefulness of science as an aid to decision making (Glantz 1979; Walters \& Maguire 1996; Garshelis 1997; Ludwig et al. 2001; Wintle et al. 2011). Within epistemic uncertainty we include structural uncertainty, parametric uncertainty, measurement error, variability, stochasticity, and information gaps.

Linguistic uncertainty arises because language is not exact. It is often ignored in conservation biology where policy and decision making play important roles, even though it is pervasive (Burgman 2005). For instance, vague and context-dependent terms or expressions can impair or even imbed implementation of political agreements. In the present study we follow the taxonomy of Regan et al. (2002) who described several sources of linguistic uncertainty relevant to applied ecology and conservation, including vagueness, ambiguity, and context de- pendence of expressions, under-specificity of statements and indeterminacy of theoretical concepts. Within linguistic subclasses we include also reasoning uncertainty (Table 1). While there are several other typologies of linguistic uncertainty (Walley \& de Cooman 2001; Lane $\&$ Maxfield 2005; Brugnach et al. 2008), the classification of Regan et al. (2002) has become widely adopted.

Recently uncertainty typologies have identified a third major source that arises from subjective judgments, differences in values and preferences, different world views, and volitional actions. In some typologies these are referred as "decision-making uncertainty" (Finkel 1990; Ascough II et al. 2008), in others as "value uncertainty" (Morgan and Henrion 1990), "volitional uncertainty" (Bedford \& Cooke 2001), "implementation error" (Harwood \& Stokes 2003), or "human uncertainty" (Maier et al. 2008). The existing terminology is heterogeneous, but all the examples describe uncertainty that arises from human beliefs, values, preferences, choices, and actions. Here, we call this type of uncertainty human decision uncertainty. The best recognized type of uncertainty within this group is subjective judgement, which is frequently used throughout decision-making processes for example parameter estimation, scenario planning or model result interpretation. Here uncertainty arises from different interpretations of data (Regan et al. 2002) and imperfectness of experts to know their limits of expertise (Burgman 2005). Values and interests of individuals are in the heart of decision making particularly in processes where sociopolitical objectives are defined and which often involve conflicts between stakeholders (Finkel 1990; Morgan \& Henrion 1990; Ascough II et al. 2008). These uncertain preferences of actors, including lack of conviction, can cause sociopolitical decisions to land far from their original scientific recommendations. On a larger scale further uncertainty may arise from the ontology of individuals: people with different cultural, religious, or political backgrounds may have variable opinions about how the relationship between humans and the Earth should be viewed, with consequent differences in opinions about conservation and climate change (Van Houtan 2010).

We note that some uncertainties are derived, others more fundamental. At the linguistic level ambiguity, vagueness, indeterminacy, and context dependence may lead to reasoning uncertainty. Measurement error is one factor underlying parametric uncertainty, and model uncertainty includes both structural and parametric components.

\section{Literature search and bibliometric analysis}

We used the Thomson Reuters' Web-of-Science ${ }^{\circledR}$ database from 1945 through to October 2011 and their 
Table 1 Types of uncertainties explained

\begin{tabular}{|c|c|}
\hline Type & Explanation \\
\hline \multicolumn{2}{|c|}{$\begin{array}{l}\text { Linguistic uncertainty: uncertainty about language and meaning of expression (Walley \& de Cooman 2001; Regan et al. } 2002 ; \text { Lane \& Maxfield 2005 } \\
\text { Brugnach et al. 2008). Linguistic uncertainty can occur without specific reference to uncertainty in text. }\end{array}$} \\
\hline Ambiguity and vagueness & $\begin{array}{l}\text { A word has more than one meaning and it is not clear which meaning } \\
\text { is intended. A word or concept allows borderline cases, or } \\
\text { different degrees of belonging to alternative concepts. }\end{array}$ \\
\hline Reasoning uncertainty & Uncertainty due to unclear argument structure. \\
\hline Context dependence & The meaning of the expression depends on context. \\
\hline Underspecificity & Overstated generality that does not extend to specific application. \\
\hline Indeterminacy & Expression requires the reader to decide on its meaning. \\
\hline
\end{tabular}

Epistemic uncertainty: incomplete knowledge; uncertainty about facts that we could know but don't know (Golding \& Rorer 1972; Regan et al. 2002; Harremoës 2003). Includes process uncertainty; uncertainty about the correct model structure and parameters (Draper 1995; Regan et al. 2002; Clark 2005). Components of model uncertainty are frequently explicitly called as such in scientific text.

Structural uncertainty

Parametric uncertainty

Variability

Stochasticity

Measurement error

Information gaps
Uncertainty about correct and adequate structural description of a model/process. Structural uncertainty is often called model uncertainty.

Uncertainty about parameter values and/or correct initial condition of a model.

Unexplained and possibly partially unknown variation in processes or process parameters across space, time, or other independent variables.

Uncertainty about specific outcome due to the influence of inherently random processes, such as determination of the sex of offspring. Inherent stochasticity can be understood and anticipated, but it cannot be reduced or eliminated. Aleatory uncertainty is a term sometimes used for inherent randomness (stochasticity) and variability.

Errors about measurements, including effects of measurement accuracy and systematic error (bias). Measurement error may underlie increased parametric uncertainty.

Severe lack of knowledge about something that probably is important for decision making. Information gaps are, e.g., unusual events that take us by surprise, or future innovations that we cannot anticipate. We know very little or nothing of their substance before they are discovered.

Human decision uncertainty: uncertainty arising from subjective human preferences, judgments, and beliefs (Morgan \& Henrion 1990; Bedford \& Cooke 2001; Ascough II et al. 2008; Maier et al. 2008).

Uncertain preferences

Uncertainties about the values, preferences, objectives, aims or goals of individuals or organizations. In this category we also included incertitude, defined as lack of conviction or certainty.

Subjective judgment

Uncertainty arising from imperfectness of expert judgment.

Ontological uncertainty

Uncertainty due to differences in the actor's ontology, that is, the actor's view about the entities that inhabit the world and the evolution of the relationships between these entities (Lane \& Maxfield 2005).

web-based search engine to identify publications relevant to conservation under climate change and uncertainty. We divided the investigation of the treatment of uncertainty into five components: climate models, economics of climate change, sociopolitical implications, ecology of species under climate change, and biodiversity con- servation under climate change. As a baseline search we used the phrase "(climate change OR global change) AND uncertainty," to which we added additional terms specific to each subtopic (Table 2). The search phrases for ecological and conservation publications were relatively complex for two reasons. First, detailed search 
Table 2 Search phrases used in literature search. Baseline phrases were included in all searches. Additional terms that were specific to each subtopic were used for literature search within each component

\begin{tabular}{ll}
\hline Baseline & (Climate change OR global change) AND uncertainty \\
Climate & AND climate model \\
Sociopolitics & AND (sociopolitical OR policy OR social OR politics) \\
Economics & AND (economics OR economical OR markets) \\
Ecology & AND (ecology OR evolution OR dispersal OR population \\
& OR metapopulation OR niche OR landscape OR \\
& connectivity) \\
Conservation & AND (biodiversity OR extinction OR ((conservation OR \\
& protection) AND (biology OR nature OR species OR \\
& habitat OR environment OR ecosystem))
\end{tabular}

phrases were needed to separate publications of these two strongly overlapping fields of research. Second, typical search words for conservation publications such as "conservation" and "protection" are not unique to biodiversity conservation, but are also found under topics such as flood protection or energy conservation. Articles from these external topics were efficiently excluded with additional search terms that confined the context to ecology and conservation. We included all primary literature articles, conference proceedings, and book chapters in the search and excluded duplicate records. PDF files for these publications were retrieved as allowed by the University of Helsinki's library access.

We mapped the occurrence of different types of uncertainties in PDF files using the PowerGREP ${ }^{\complement}$ search tool (Just Great Software Co. Ltd, Rawai Phuket, Thailand). Within each subtopic we counted the number of occurrences of each uncertainty type inside the full-text PDF, using the search terms listed in Table 1 together with their potential synonyms, and allowing certain common variation in the terminology (see Appendix S1). We found that terminology and the usage of terms can be ambiguous and varies between subtopics. Therefore, each search result was individually evaluated for meaning and context, to verify that the occurrence corresponded to the type of uncertainty of interest. Occurrences were excluded of terms that were considered irrelevant, or which were in the literature list of a publication.

\section{Evaluation of conservation strategies under climate change}

Characterizing potential sources of uncertainty and their implications for conservation strategies may provide useful insight into the prospects for successful conservation outcomes under climate change. Heller \& Zavaleta (2009) identified from past literature 113 conservation strategies that had been recommended as adaptation measures to climate change. For illustration, we created a schematic figure that links the 21 most often recommended strategies from Heller \& Zavaleta (2009) to processes and factors relevant to conservation under climate change, with the purpose of understanding the position and influence paths of each strategy. We then evaluated potential uncertainties associated with these conservation strategies through a short questionnaire that was distributed to 20 researchers working with conservation issues. The 20 scientists were identified from the professional networks of the authors. The purpose of the questionnaire was to sample perceptions of the relative magnitudes of different types of uncertainty, held by scientists working in ecology and conservation biology. We note that the answers collected for the study are a nonrandom sample and should therefore be seen as an illustrative example rather than a comprehensive overview of scientific opinions. For each strategy we asked participants to assess on a scale of lowmedium-high (1) the extent of vague statements and unclear terms (linguistic uncertainty), (2) the general level of knowledge and availability of data for implementation of the strategy (epistemic uncertainty), and (3) evidence of value-based, subjective decision making (human decision uncertainty). To calculate general metrics the answers were converted into numerical values (low $=1$, low $/$ medium $=1.5$, medium $=2$, medium $/$ high $=2.5$, high $=3$, respectively) .

Finally, we analyzed the literature of one of the conservation strategies, species translocations, in more detail from the perspective of the three classes of uncertainty employed in this work. Note that this type of evaluation could potentially be done for all of the strategies listed in Heller \& Zavaleta (2009), but such a task is beyond the scope of this work. We stress that our evaluations are not exhaustive, but serve the purpose of illustrating how different sources of uncertainty could be characterized to estimate the potential usefulness of conservation strategies and to assist decision making.

\section{Results}

\section{Treatment of uncertainty in scientific literature}

The literature search found 65,852 publications under the topic of climate change or global change research, out of which 3,494 (5.3\%) included the word "uncertainty" in the title, abstract, or key words. The volume of uncertainty literature has been increasing rapidly, with 113 articles published by 1995 or earlier $(2.8 \%$ of all articles focusing on climate/global change), 330 articles (4.7\%) in 1996-2000, 626 articles (5.0\%) in 2001-2005, 1,802 articles $(5.5 \%)$ in $2006-2010$, and 623 articles $(6.8 \%)$ in 2011 by November. 
Table 3 Number of publications found in the literature research, proportion of available PDF files, and overlap between components. The numbers in column titles show the total number of publications found in each literature component ( $n$ ) and the respective proportion of PDF files that was available to us (\%). In the table, numbers above the diagonal show the number of publications shared by any two components. Numbers below the diagonal show the percentage of each literature component that is shared by other components, with the first number read as row shared by column and the second vice versa. (Sociopolitical publications shared $34 / 865=3.9 \%$ of publications with the climate model component, and so on.)

\begin{tabular}{|c|c|c|c|c|c|}
\hline & Climate & Sociopolitics & Economics & Ecology & Conservation \\
\hline$n$ & 314 & 865 & 186 & 543 & 297 \\
\hline Available (\%) & $94 \%$ & $87 \%$ & $89 \%$ & $91 \%$ & $94 \%$ \\
\hline Climate & & 34 & 4 & 28 & 6 \\
\hline Sociopolitics & $3.9 \% / 10.8 \%$ & & 131 & 116 & 70 \\
\hline Economics & $2.2 \% / 1.3 \%$ & $70.0 \% / 15.1 \%$ & & 14 & 11 \\
\hline Ecology & $5.2 \% / 8.9 \%$ & $21.4 \% / 13.4 \%$ & $2.6 \% / 7.5 \%$ & & 134 \\
\hline Conservation & $2.0 \% / 1.9 \%$ & $23.6 \% / 8.1 \%$ & $3.7 \% / 3.7 \%$ & $45.1 \% / 24.7 \%$ & \\
\hline
\end{tabular}

Five searches of specific subtopics identified a total of 1,735 unique publications, some of which appeared in two or more subtopics. These Web-of-Science searches resulted in 314, 865, 186, 548, and 297 publications identified for the climatic, sociopolitical, economic, ecological, and conservation components, respectively. While search phrases were chosen to target separate subtopics, there was overlap between them because many publications span multiple subtopics. For example, publications found by the sociopolitical search were frequently shared by other sets of search results. Table 3 summarizes results of the literature searches, including overlap between subsets. Overall, the degrees of overlap between subtopics varied from $1.3 \%$ (between climate modeling and economics research) to $70 \%$ (most economics papers included a sociopolitical component). The mean overlap between sets was $13.9 \%$.

The occurrence of different types of uncertainty (Table 1) in the five literature components is summarized in Table 4 and Figures 1 and Sl. We found that the different kinds of uncertainty are unevenly represented with the majority of studies focusing on various forms of epistemic uncertainty. Inherent variability of processes was the most commonly mentioned term followed by stochasticity in all fields except economics. In the comparatively small economics set, stochasticity occurred most frequently, but almost equal occurrences of other epistemic uncertainties (structural, parametric, variability) were mentioned. Linguistic uncertainty was not well recognized in general, and the majority of its classes were found only in studies that included elements of socialpolitical sciences. Human decision uncertainty, including ontological uncertainty, uncertain preferences, and subjective judgment was pervasive, but generally patchily treated. In particular, uncertainty about values (preferences, goals, objectives, aims) was mentioned very infrequently, even though it would seem that such uncertainty is present whenever decisions are made.

\section{Influence paths of conservation strategies}

Figure 2 illustrates how conservation strategies link to processes and factors relevant to conservation under climate change. We have separated processes and factors into those that humans can influence directly (sociopolitics, economics, and conservation priorities) and those we can influence only indirectly (ecological and climatic processes). First, and most importantly, the schematic emphasizes that different conservation strategies have very different impact points on processes and factors. Second, the effects of biodiversity conservation efforts have direct impacts on populations, and indirect influences mediated via human impacts on landscape structure.

Some conservation strategies, such as increasing number of reserves, buffering conservation areas, and practicing adaptive management, have relatively direct and immediate influence paths-these strategies can be viewed as implementation strategies. In contrast, strategies such as studying responses of species to climate change and developing improved modeling and analysis capacity, improve our understanding about ecological and climatic processes. The impact of these strategies is indirect and relatively slow, mediated by changes in sociopolitical preferences and conservation objectives, influencing the way conservation is ultimately implemented. The figure also emphasizes the central importance of understanding and managing economic considerations in conservation planning. Note that Figure 2 should primarily be seen as a vehicle for structuring thinking around conservation strategies, not as a true or complete depiction of processes relevant to conservation biology under climate change. Ultimately, all these processes and factors are linked. We 
Table 4 Counts and percentiles of publications mentioning different types of uncertainties in each literature component. The numbers in column titles (n) give the total number of articles found for each component. Columns show the count of articles mentioning a particular type of uncertainty inside the article text; the numbers in parentheses are the same information expressed as percentage of publications.

\begin{tabular}{|c|c|c|c|c|c|}
\hline & $\begin{array}{l}\text { Climate } \\
n=314\end{array}$ & $\begin{array}{c}\text { Sociopolitics } \\
n=865\end{array}$ & $\begin{array}{c}\text { Economics } \\
n=186\end{array}$ & $\begin{array}{l}\text { Ecology } \\
n=543\end{array}$ & $\begin{array}{c}\text { Conservation } \\
n=297\end{array}$ \\
\hline \multicolumn{6}{|l|}{ Linguistic uncertainty } \\
\hline Ambiguity and vagueness & $16(5.1 \%)$ & $154(17.8 \%)$ & $32(17.2 \%)$ & $45(8.3 \%)$ & $31(10.4 \%)$ \\
\hline Reasoning uncertainty & $4(1.3)$ & $12(1.4)$ & $1(0.5)$ & $9(1.7)$ & $4(1.3)$ \\
\hline Context dependence & $2(0.6)$ & $4(0.5)$ & $7(3.8)$ & $11(2.0)$ & $6(2.0)$ \\
\hline Underspecificity & $8(2.5)$ & $14(1.6)$ & $2(1.1)$ & $8(1.5)$ & $3(1.0)$ \\
\hline Indeterminacy & $3(1.0)$ & $51(5.9)$ & $10(5.4)$ & $11(2.0)$ & $4(1.3)$ \\
\hline \multicolumn{6}{|l|}{ Epistemic uncertainty } \\
\hline Structural uncertainty & $148(47.1)$ & $162(18.7)$ & $52(28.0)$ & $139(25.6)$ & $56(18.9)$ \\
\hline Parametric uncertainty & $81(25.8)$ & $167(19.3)$ & $50(25.9)$ & $96(17.7)$ & $39(13.1)$ \\
\hline Variability & $252(80.3)$ & $340(39.3)$ & $49(26.3)$ & $312(57.5)$ & $155(52.2)$ \\
\hline Stochasticity & $58(18.5)$ & $179(20.7)$ & $61(32.8)$ & $124(22.8)$ & $67(22.6)$ \\
\hline Measurement error & $173(55.1)$ & $222(25.7)$ & $35(18.8)$ & $202(37.2)$ & $111(37.4)$ \\
\hline Information gap & $2(0.6)$ & $14(1.6)$ & $3(1.6)$ & $13(2.4)$ & $11(3.7)$ \\
\hline \multicolumn{6}{|l|}{ Human decision uncertainty } \\
\hline Subjective judgment & $10(3.2)$ & $92(10.6)$ & $23(12.4)$ & $32(5.9)$ & $18(6.1)$ \\
\hline Uncertain preferences & $1(0.3)$ & $9(1.0)$ & $4(2.2)$ & $1(0.2)$ & $1(0.3)$ \\
\hline Ontological uncertainty & $0(0.0)$ & $13(1.5)$ & $0(0.0)$ & $3(0.6)$ & $0(0.0)$ \\
\hline
\end{tabular}

have shown only those linkages that we considered being relatively important. The longer (the less direct, or the more intervening nodes along) the influence path of a strategy, the larger are the associated uncertainties and the longer it takes for the strategy to produce results.

\section{Evaluation of proposed conservation strategies against types of uncertainty}

Despite the putatively similar backgrounds and motivations of our questionnaire respondents, there is considerable variation in how different conservation strategies are seen to be impaired by different types of uncertainty (Table 5). Nevertheless, some consistent differences and convincing patterns emerged from this exercise: First, based on our respondents' opinions the highest uncertainties arise from human decisions whereas linguistic uncertainty was most often ranked as lowest. Second, respondents seem to agree most on their high uncertainty scores (mean of standard deviations $=0.58$ ) and disagree on uncertainties that on average received intermediate (0.71) and low (0.67) scores. Third, if conservation strategies are ranked according to their overall average uncertainty value, the order of strategies is notably different from the original ranking of Heller $\&$ Zavaleta (2009). Strategies that are most often recommended in conservation literature as adaptation measures under climate change, such as integration of climate change to all planning exercises, increasing connectivity and miti- gation of other threats, are according to our respondents associated with high uncertainties in several or all categories (Table 5). Direct actions aiming at conserving additional areas (either as protected areas or buffer zones) were seen as least uncertain strategies, closely followed by strategies aimed to improve our understanding of climate change impacts (monitoring programs and studying ecological consequences of climate change).

\section{Translocating species under climate change-looking into the details}

Under climate change, many species face challenges if they are to follow their moving climate regimes, especially species with limited dispersal abilities in regions in which habitat is becoming increasingly fragmented (Lawler \& Olden 2011). Translocation (or assisted migration) has been suggested as a strategy to aid species to overcome this challenge (Heller \& Zavaleta 2009) and has been hotly debated in recent years (Hewitt et al. 2011).

The debate has focused on epistemic issues, including the potential impacts of the translocated species on destination biota, the inherent uncertainty in predicting the invasiveness of species and the spread of parasites and pathogens (Ricciardi \& Simberloff 2009). Additional significant uncertainty stems from technical aspects-how many and what types of individuals to move, when and how to move them and between which locations (Hulme 2005; McLachlan et al. 2007). An underlining source 

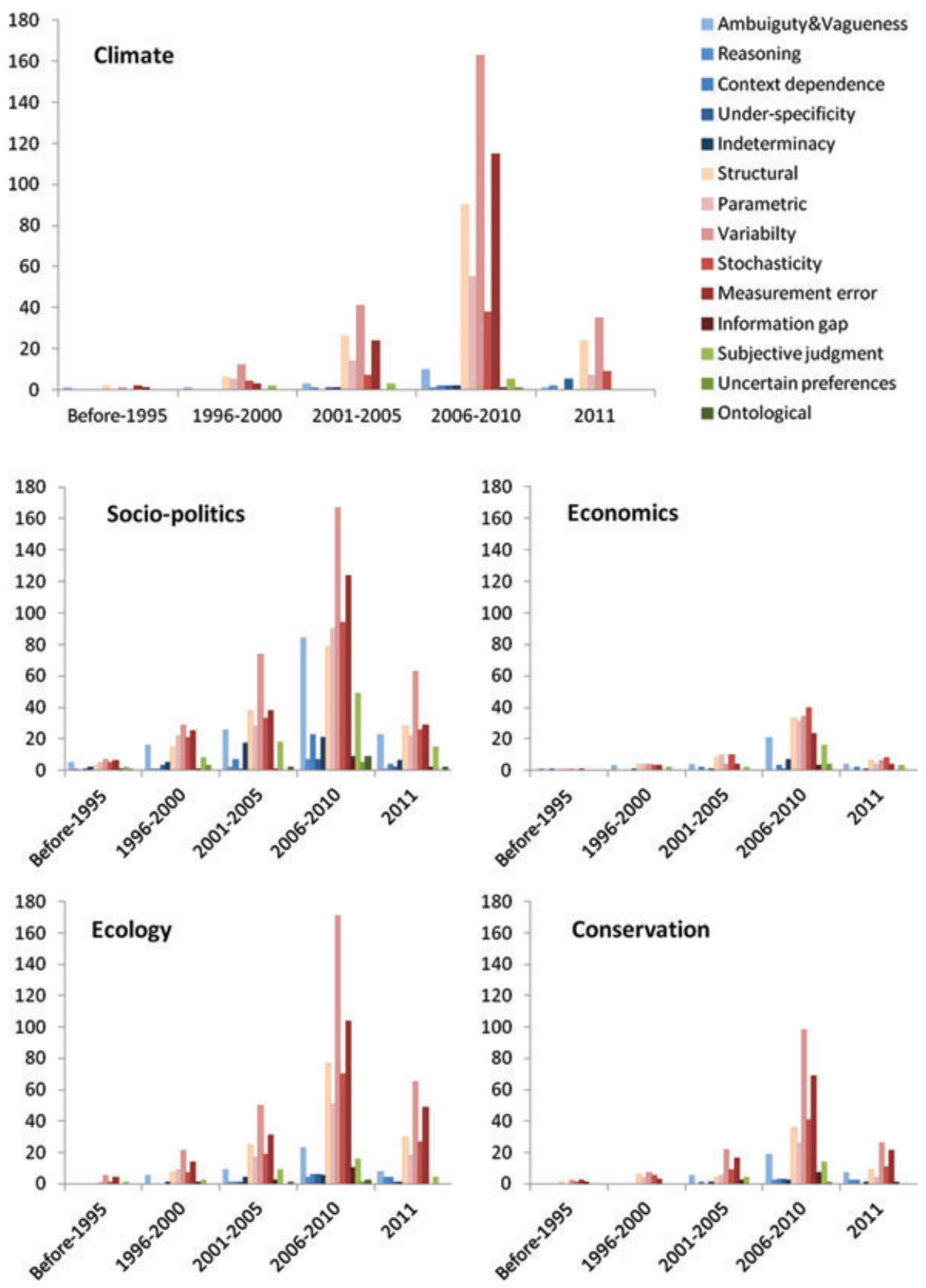

Figure 1 Development through time of the numbers of publications mentioning types of uncertainties in each literature component. Note that there is a general increasing trend in scientific literature under the topic of climate change and uncertainty, which partly explains the increase in

the number of publications shown in this figure (see text). Proportion of publications mentioning different types of uncertainty is shown in the Supplementary Figure $\mathrm{S} 1$.

of uncertainty is also the question of predicting future changes in climate, its impacts on populations and how areas become suitable or unsuitable for the species being translocated (Hagerman et al. 2010).

Problems of epistemic uncertainty tend to be acknowledged in the assisted migration literature. Hewitt et al.

(2011) pointed out that the major debate around biological uncertainties is driven by a lack of empirical case studies and severe taxonomic and geographical bias (toward plants and western countries), which hinders the establishment of general consensus. Additional research can address these questions, but is unlikely to 


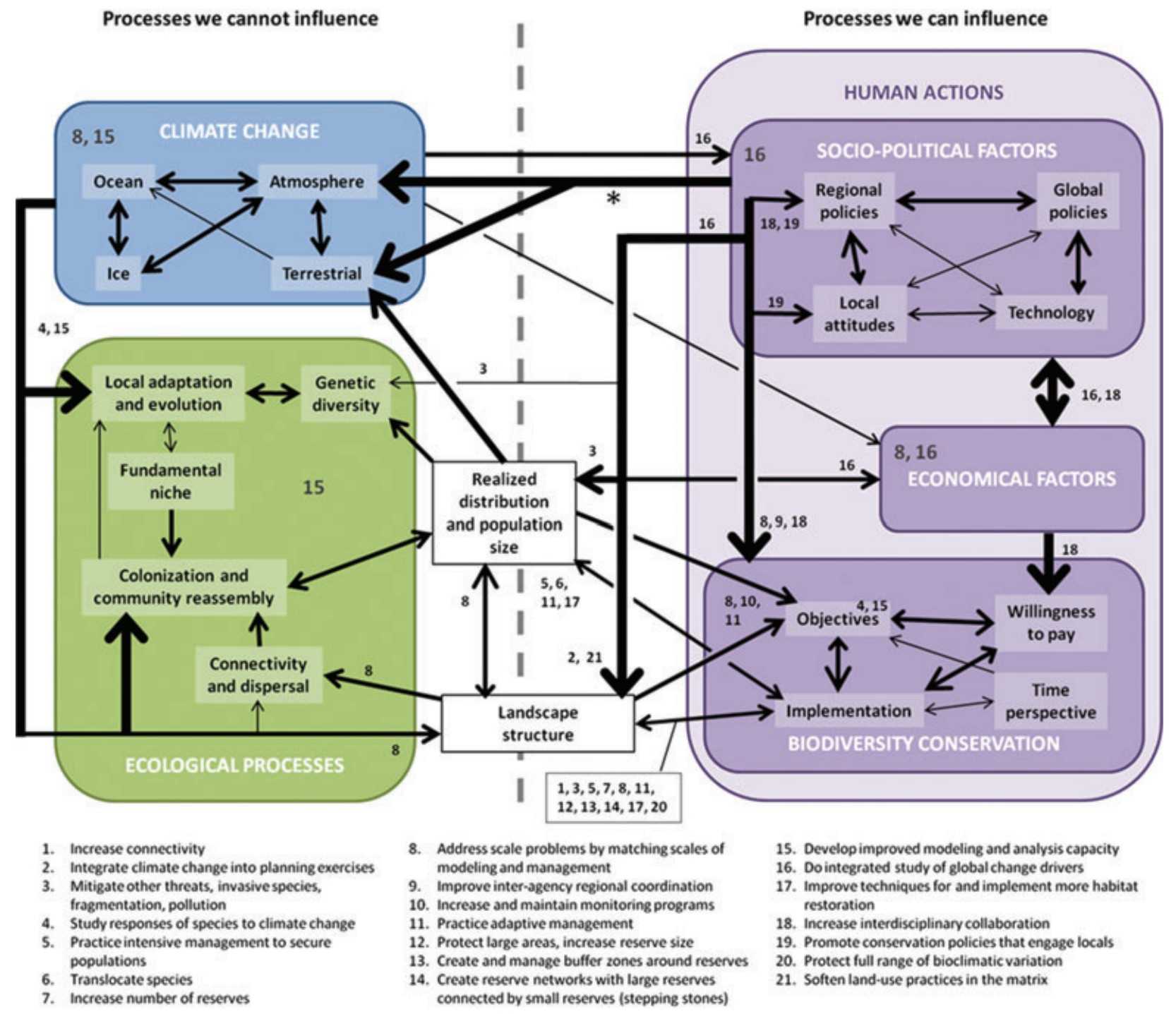

Figure 2 Major factors and processes relevant to biodiversity conservation under climate change; the thickness of an arrow indicates relative strength of effect and asymmetric relations are indicated by arrow heads of different sizes. The numbers refer to the top 21 strategies of Heller \& Zavaleta (2009), given at the bottom of the figure; the impact point of each strategy is marked by the position of the strategy in the figure. Climate change mitigation, mentioned frequently in other conservation literature, is not included in Heller \& Zavaleta's (2009) top 21 conservation strategies; we have marked the location of that strategy by $(*)$.

will inevitably be coupled with high uncertainty of future events, which cannot be entirely solved or removed. Hence, many of the epistemic uncertainties are well understood, but some are more difficult to address than others.

For large parts of the assisted migration literature, linguistic uncertainty is not considered to be a major issue. Vitt et al. (2009) raise the problem of vague terminology that does not separate between translocations conducted for economic or conservation reasons. Context dependence and underspecificity may also arise in 
Table 5 Conservation strategies (adapted from Heller \& Zavaleta 2009) evaluated against uncertainty and operational characteristics of the strategy. The table includes the first 21 strategies ranked from first to ninth based on the number of publications recommending that strategy. The number of the strategy is the same as in Figure 2 . The uncertainty values give mean and standard deviation calculated from the questionnaire (low $=1$, low $/ \mathrm{medium}=$ 1.5 , medium $=2$, medium/high $=2.5$, high $=3$ ). The mean values were further divided into three equal intervals, light green colouring denoting values with a low (1.0-1.6) mean uncertainty score, and light red indicating high (2.4-3.0) mean uncertainty scores. The mean total uncertainty is the mean across the three uncertainty classes. The influence path defines whether each strategy can be seen as a direct (DA) or indirect (IA) action or as something that produces information that can influence conservation decisions (increasing information, II)

\begin{tabular}{|c|c|c|c|c|c|c|c|}
\hline & Strategy & & & Uncertainty & & & \\
\hline \# & Description & path & Linguistic & Epistemic & Human & uncertainty & Heller \& Zavaleta (2009) \\
\hline 12 & Protect large areas, increase reserve size & DA & $1.4 \pm 0.60$ & $1.6 \pm 0.71$ & $\mathbf{2 . 0} \pm 0.77$ & 1.7 & 7 \\
\hline 7 & Increase number of reserves* & DA & $1.3 \pm 0.61$ & $1.8 \pm 0.64$ & $2.2 \pm 0.82$ & 1.8 & 5 \\
\hline 13 & $\begin{array}{l}\text { Create and manage buffer zones around } \\
\text { reserves }\end{array}$ & DA & $1.4 \pm 0.56$ & $1.9 \pm 0.52$ & $2.0 \pm 0.75$ & 1.8 & 8 \\
\hline 10 & Increase and maintain monitoring programs & II & $1.7 \pm 0.75$ & $1.8 \pm 0.62$ & $2.3 \pm 0.64$ & 1.9 & 7 \\
\hline 4 & Study responses of species to climate change & II & $1.6 \pm 0.76$ & $2.4 \pm 0.64$ & $2.1 \pm 0.82$ & 2.0 & 4 \\
\hline 14 & $\begin{array}{l}\text { Create reserve networks with large reserves } \\
\text { connected by small reserves (stepping } \\
\text { stones) }\end{array}$ & DA & $1.7 \pm 0.8$ & $\mathbf{2 . 2} \pm 0.69$ & $\mathbf{2 . 3} \pm 0.70$ & 2.0 & 9 \\
\hline 17 & $\begin{array}{l}\text { Improve techniques for and implement more } \\
\text { habitat restoration }\end{array}$ & DA & $1.8 \pm 0.71$ & $2.4 \pm 0.49$ & $2.2 \pm 0.67$ & 2.1 & 9 \\
\hline 9 & Improve interagency regional coordination & $\mathrm{IA}$ & $2.2 \pm 0.64$ & $1.6 \pm 0.67$ & $2.6 \pm 0.58$ & 2.1 & 6 \\
\hline 18 & Increase interdisciplinary collaboration & IA & $2.5 \pm 0.60$ & $1.8 \pm 0.64$ & $\mathbf{2 . 3} \pm 0.79$ & 2.2 & 9 \\
\hline 6 & Translocate species & DA & $1.5 \pm 0.71$ & $2.6 \pm 0.63$ & $2.6 \pm 0.60$ & 2.2 & 4 \\
\hline 8 & $\begin{array}{l}\text { Address scale problems by matching scales of } \\
\text { modeling and management }\end{array}$ & IA & $1.9 \pm 0.88$ & $2.4 \pm 0.69$ & $2.4 \pm 0.69$ & 2.2 & 6 \\
\hline 19 & $\begin{array}{l}\text { Promote conservation policies that engage } \\
\text { locals }\end{array}$ & IA & $2.3 \pm 0.80$ & $\mathbf{2 . 0} \pm 0.55$ & $2.6 \pm 0.46$ & 2.3 & 9 \\
\hline 20 & Protect full range of bioclimatic variation & DA & $\mathbf{2 . 2} \pm 0.81$ & $2.4 \pm 0.74$ & $2.3 \pm 0.73$ & 2.3 & 9 \\
\hline 5 & $\begin{array}{l}\text { Practice intensive management to secure } \\
\text { populations }\end{array}$ & DA & $\mathbf{2 . 3} \pm 0.72$ & $2.2 \pm 0.50$ & $\mathbf{2 . 4} \pm 0.59$ & 2.3 & 4 \\
\hline 15 & $\begin{array}{l}\text { Develop improved modeling and analysis } \\
\text { capacity }\end{array}$ & ॥ & $2.4 \pm 0.65$ & $2.4 \pm 0.65$ & $2.2 \pm 0.75$ & 2.3 & 9 \\
\hline 3 & $\begin{array}{l}\text { Mitigate other threats, invasive species, } \\
\text { fragmentation, pollution }\end{array}$ & DA & $2.2 \pm 0.75$ & $2.3 \pm 0.64$ & $2.6 \pm 0.56$ & 2.4 & 3 \\
\hline 1 & Increase connectivity & DA & $2.6 \pm 0.60$ & $2.2 \pm 0.61$ & $2.4 \pm 0.69$ & 2.4 & 1 \\
\hline 11 & Practice adaptive management & DA & $2.2 \pm 0.75$ & $2.5 \pm 0.65$ & $2.6 \pm 0.5$ & 2.4 & 7 \\
\hline 21 & Soften land-use practices in the matrix & DA & $\mathbf{2 . 4} \pm 0.59$ & $\mathbf{2 . 2} \pm 0.75$ & $2.8 \pm 0.41$ & 2.5 & 9 \\
\hline 16 & Do integrated study of global change drivers & II & $2.6 \pm 0.67$ & $2.8 \pm 0.41$ & $2.4 \pm 0.67$ & 2.6 & 9 \\
\hline 2 & $\begin{array}{l}\text { Integrate climate change into planning } \\
\text { exercises }\end{array}$ & IA & $2.6 \pm 0.63$ & $2.9 \pm 0.24$ & $2.9 \pm 0.33$ & 2.8 & 2 \\
\hline
\end{tabular}

* Assuming reasonable increase in total reserve area.

defining locations or movement (McLachlan et al. 2007): How far does a manager need to move an individual before it is considered a translocation event? Nevertheless, in comparison with other types of uncertainties, furthering discussion and clarifying definitions were seen as a reasonably straightforward solution (Hewitt et al. 2011).

Species translocations involve important sources of $h u$ man decision uncertainty. The most obvious is the choice of species to be translocated. Inevitably this will be a subjective decision, which can be driven by different values, opinions, or even economic interests (Hayward 2009; Hagerman et al. 2011; Lawler \& Olden 2011). But significant uncertainties arise also from the larger context: for example, translocations are seen by many as "unnatural" or "unethical" interventions (Vitt et al. 2010) that can contradict other conservation objectives (i.e., translocating a threatened species vs. protecting the natural state of destination biota, McLachlan et al. 2007). Such views may lead to resistance of the strategy at different levels of the decision-making process. 
Translocation attempts may also fail due to lack of local social support (Parker 2008), which is typical for reintroductions of large predators, or if there are political or legislative obstacles that prevent intergovernmental or interagency cooperation (Lawler \& Olden 2011). Several sources of human decision uncertainty are named and discussed in the assisted migration literature, and notably many are seen as major barriers to more productive discussion regarding the success of this strategy (Hewitt et al. 2011 ; Lawler 8 Olden 2011). Yet, concrete suggestions of how to address these uncertainties are few and generally aim at improving our understanding of the processes, or widening the discussion and involving audiences beyond the scientific community (Hagerman et al. 201 1; e.g., Hayward 2009).

Based on the rapidly accumulating literature, the relative magnitudes of different types of uncertainty support our questionnaire results in the sense that for this particular strategy, linguistic uncertainties are considered to be less important than epistemic and human decision uncertainties (Table 5). Translocating species is clearly a direct conservation action (Figure 2 ), but as revealed by this evaluation, it is also dependent on several epistemic and sociopolitical factors that can have far more complex and indirect influence paths.

\section{Discussion}

Uncertainty influences decision making as usually decision makers prefer reliable outcomes over very uncertain ones (Burgman 2005; Game et al. 2011; Polasky et al. 2011; Wintle et al. 2011). There are clear differences in the representation of types of uncertainty between fields of science indicating that the fundamentally different assumptions that appear conventional in one area, are often ignored in another; linguistic and human decision uncertainties are better acknowledged in sociopolitical and economic sciences whereas epistemic uncertainties dominate the literature in natural and physical sciences, particularly in climate research. These differences reflect the position of each type of uncertainty in the conservation decision-making process. Scientists in sociopolitical and economic fields work more frequently with issues related to human perceptions and implementation of policies (Ascough II et al. 2008; Swart et al. 2009). Hence, studies in these domains tend to be relatively more explicit about linguistic and decision uncertainties, albeit that concepts are not necessarily well defined. Natural sciences, on the other hand, are more focused on observing patterns in nature, understanding causalities, and making recommendations based on verified theories (Ludwig et al. 2001). Treatments of linguistic and human decision uncertainties occur less frequently in them. Nevertheless, underrepresentation of human decision uncertainty in natural sciences publications is striking given how widely expert judgments are used in data selection, model building, and parameterization, and how relatively sensitive these topics are to biases, motivations, values, and attitudes (Burgman 2005). The differences between fields of research highlight the importance of cross-disciplinary studies for efficient decision making.

We note that the literature analysis presented here is inevitably limited by potential biases in its search methods. Some of our search terms, such as structural uncertainty or parametric uncertainty are well known and are used routinely to indicate uncertainty. Other terms can be found by text search only in a limited manner. For example vagueness is something that cannot typically be found by searching for the word "vague." Full analysis of vagueness would require examination of every sentence of text to identify words or expressions that allow borderline cases-a task that is for practical purposes impossible for hundreds of articles. The same caveat may apply to other subtypes of linguistic and human decision uncertainty: many of these terms occur without explicit recognition and are used in text typically only when the potential for such uncertainty is explicitly acknowledged. Consequently, the numbers we report in Tables 3 and 4 are informative for a search term between different literature components and time periods, but the numbers are less than fully comparable between different types of uncertainty. Even so, it is clear that human decision and linguistic uncertainties are infrequently and inconsistently treated, compared to epistemic uncertainty. Much of quantitative science is in fact about the reduction of various forms of epistemic uncertainty, i.e., lack of knowledge about something we could know about, and it therefore is not surprising to find epistemic uncertainty relatively widely treated.

Our exploration of conservation strategies in terms of their inherent uncertainties reveals how seemingly reliable strategies may be subject to considerable unacknowledged uncertainty. Recommendations from the natural sciences often ignore human decision and linguistic uncertainty, overlooking communication between stakeholders and socioeconomic consequences, potentially rendering many such recommendations socially naïve and exposing them to significant social backlashes (Ludwig et al. 2001; Wintle et al. 2011). Mental models strongly influence the actions of people, and awareness of human decision uncertainty may help anticipate conflicts of interest and competing aspirations (Walters \& Maguire 1996; Ascough II et al. 2008). Linguistic uncertainty may compromise conservation success by generating actions that are contrary to the intended recommendation, or that are unenforceable (Ludwig et al. 
2001). High epistemic uncertainty impedes quantitative decision making, leading to reliance on subjective judgment. Systematic discussion about aims and evaluation criteria may reduce human decision uncertainty (e.g., Fernandes et al. 1999), although it may not be completely removed due to human behavior and different world views of different actors. Definition of concepts and their interrelations can reduce linguistic uncertainty significantly. Epistemic uncertainty can be reduced, albeit not completely removed, by improvements in data and its analysis.

However, it should also be noted that in conservation planning, both scientists and decision makers are frequently required to make recommendations and decisions before the full story is known (Dawson et al. 2011; Polasky et al. 2011). Sometimes uncertain strategies may be the only way to address problems and challenges faced in the conservation arena. The focus of this work is to improve our understanding of uncertainty, not to define which conservation strategies are most needed in climate change adaptation. Evaluating conservation strategies in terms of the uncertainty components outlined in Table 1, either quantitatively or qualitatively as illustrated in this work, will improve decision makers' understanding of the characteristics of the strategy being considered. Linking strategies to process paths such in Figure 2 further communicates the influence linkages and points of impact of strategies as well as their associated uncertainties. Such an evaluation can also lead to identification of research needs to reduce sources of uncertainty inherent in conservation strategies.

Our review of literature shows that there remains scope for improvements when accounting for uncertainty in decision making. First, there is a critical need for a common terminology for linguistic and human decision uncertainty, in conservation science and in other disciplines (Morgan \& Henrion 1990; Charles 1998; Harwood \& Stokes 2003; Walker et al. 2003). Second, despite the recent advances in accounting for the uncertainties of human input (e.g. modeling human behavior [Anderies 2000; Peterson 2000], and multiple criterion decision analysis [MCDA] [Roy 1999; Linkov et al. 2006]) there remains a need to address these uncertainties more explicitly, as they may outweigh technical and scientific drivers (Morgan \& Dowlatabadi 1996; Ascough II et al. 2008) (Table 5). Third, methodologies that transparently facilitate decision making under uncertainty need to be adopted. There exist analytical frameworks that account for uncertainty, and evaluate the likelihoods of outcomes and their possible consequences (Arvai 2007; Raiffa et al. 2007), but these tools are still rarely implemented in conservation planning and decision making (Langford et al. 2011; Wintle et al. 2011).

\section{Acknowledgments}

A.M. and H.K. thank the Academy of Finland centre of excellence programme 2006-2011. A.M. acknowledges the ERC-StG project GEDA (grant 260393) and H.K. acknowledges the EU-FP7 RESPONSES-244092 and LUOVA Graduate School for financial support. We thank A. Kukkala and J. Kuusterä for assistance with the compilation of the literature database. Our sincere thanks to A. Arponen, M. Cabeza, A. Cameron, Y.E. Chee, E. Di Minin, J. Eklund, R. Garcia, J. Kool, J. Laitila, J. Leathwick, J. Lehtomäki, C. MacAlpine, M. Maron, L. Meller, F. Montesino Pouzols, N. Strange, C.D. Thomas, and L. Zupan for their assistance on the strategy evaluation. We also thank four anonymous referees for their comments on earlier versions of this work.

\section{Supporting Information}

Additional Supporting Information may be found in the online version of this article at the publisher's web site:

Appendix S1: List of uncertainty types and their respective regex search terms

Figure S1: Development in the proportion of publications mentioning different types of uncertainty through time

\section{Literature cited}

Anderies, J.M. (2000) On modeling human behavior and institutions in simple ecological economic systems. Ecol. Econ., 35, 393-412.

Arvai, J. (2007) Rethinking of risk communication: lessons from the decision sciences. Tree Genet. Genomes, 3, 173-185.

Ascough, II J.C., Maier, H.R., Ravalico, J.K. \& Strudley, M.W. (2008) Future research challenges for incorporation of uncertainty in environmental and ecological decision-making. Ecol. Modell., 219, 383-399.

Bedford, T. \& Cooke, R.M. (2001) Probabilistic risk analysis: foundations and methods. Cambridge University Press, Cambridge, UK.

Bellard, C., Bertelsmeier, C., Leadley, P., Thuiller, W. \& Courchamp, F. (2012) Impacts of climate change on the future of biodiversity. Ecol. Lett., 15, 365-377.

Brugnach, M., Dewulf, A., Pahl-Wostl, C. \& Taillieu, T. (2008) Toward a relational concept of uncertainty: about knowing too little, knowing too differently, and accepting not to know. Ecol. Soc., 13, 30. [Online] URL. Available from: http://www.ecologyandsociety.org/vol13/iss32/art30/.

Burgman, M.A. (2005) Risks and decisions for conservation and environmental management. Cambridge University Press, Cambridge, UK.

Carvalho, S.B., Brito, J.C., Crespo, E.J. \& Possingham, H.P. (2010) From climate change predictions to actions 
- conserving vulnerable animal groups in hotspots at a regional scale. Global Change Biol., 16, 3257-3270.

Charles, A.T. (1998) Living with uncertainty in fisheries: analytical methods, management priorities and the Canadian groundfishery experience. Fish. Res., 37, 37-50.

Clark, J.S. (2005) Why environmental scientists are becoming Bayesians. Ecol. Lett., 8, 2-14.

Dawson, T.P., Jackson, S.T., House, J.I., Prentice, I.C. \& Mace, G.M. (2011) Beyond predictions: biodiversity conservation in a changing climate. Science, 332, 53-58.

Deser, C., Phillips, A., Bourdette, V. \& Teng, H. (2012) Uncertainty in climate change projections: the role of internal variability. Climate Dyn., 38, 527-546.

Draper, D. (1995) Inference and hierarchical modeling in the social sciences. J. Educ. Behav. Stat., 20, 115-147.

Fernandes, L., Ridgley, M.A. \& van't Hof, T. (1999) Multiple criteria analysis integrates economic, ecological and social objectives for coral reef managers. Coral Reefs, 18, 393-402.

Finkel, A.M. (1990) Confronting uncertainty in risk management: a guide for decision-makers. Resources for the Future Press, Center for Risk Management, Washington, D.C., USA.

Game, E.T., Lipsett-Moore, G., Saxon, E., Peterson, N. \& Sheppard, S. (2011) Incorporating climate change adaptation into national conservation assessments. Global Change Biol., 17, 3150-3160.

Garshelis, D.L. (1997) Sea otter mortality estimated from carcasses collected after the Exxon Valdez oil spill. Conserv. Biol., 11, 905-916.

Glantz, M.H. (1979) Science, politics and economics of the Peruvian anchoveta fishery. Mar. Policy, 3, 201-210.

Golding, S.L. \& Rorer, L.G. (1972) Illusory correlation and subjective judgment. J. Abnormal Psychol., 80, 249-260.

Hagerman, S., Dowlatabadi, H., Chan, K.M.A. \& Satterfield, T. (2011) Integrative propositions for adapting conservation policy to the impacts of climate change. Global Environ. Change, 20, 351-362.

Hagerman, S., Dowlatabadi, H., Satterfield, T. \& McDaniels, T. (2010) Expert views on biodiversity conservation in an era of climate change. Global Environ. Change, 20, 192-207.

Harremoës, P. (2003) The need to account for uncertainty in public decision making related to technological change. Integr. Assess., 4, 18-25.

Harwood, J. \& Stokes, K. (2003) Coping with uncertainty in ecological advice: lessons from fisheries. Trends Ecol. Evol., 18, 617-622.

Hayward, M. (2009) Conservation management for the past, present and future. Biodiver. Conserv. 18, 765-775.

Heller, N.E. \& Zavaleta, E.S. (2009) Biodiversity management in the face of climate change: a review of 22 years of recommendations. Biol. Conserv. 142, 14-32.

Hewitt, N., Klenk, N., Smith, A.L. et al. (2011) Taking stock of the assisted migration debate. Biol. Conserv. 144, 2560-2572.

Hulme, P.E. (2005) Adapting to climate change: is there scope for ecological management in the face of a global threat? $J$. Appl. Ecol., 42, 784-794.
Knight, F.H. (1921) Risk, uncertainty and profit. Houghton Mifflin Company, Boston and New York.

Lane, D.A. \& Maxfield, R.R. (2005) Ontological uncertainty and innovation. J. Evol. Econ., 15, 3-50.

Langford, W.T., Gordon, A., Bastin, L., Bekessy, S.A., White, M.D. \& Newell, G. (2011) Raising the bar for systematic conservation planning. Trends Ecol. Evol., 26, 634-640.

Lawler, J.J. \& Olden, J.D. (2011) Reframing the debate over assisted colonization. Frontiers Ecol. Environ., 9, 569-574.

Linkov, I., Satterstrom, F.K., Kiker, G., Batchelor, C., Bridges, T. \& Ferguson, E. (2006) From comparative risk assessment to multi-criteria decision analysis and adaptive management: recent developments and applications. Environ. Int., 32, 1072-1093.

Ludwig, D., Mangel, M. \& Haddad, B. (2001) Ecology, conservation, and public policy. Ann. Rev. Ecol. Syst., 32, 481-517.

Maier, H.R., Ascough Ii, J.C., Wattenbach, M., Renschler, C.S., Labiosa, W.B. \& Ravalico, J.K. (2008) Chapter five uncertainty in environmental decision making: issues, challenges and future directions. Dev. Integr. Environ. Assess., 3, 69-85.

McDonald-Madden, E., Runge, M.C., Possingham, H.P. \& Martin, T.G. (2011) Optimal timing for managed relocation of species faced with climate change. Nat. Climate Change, $\mathbf{1}$, 261-265.

McLachlan, J.S., Hellmann, J.J. \& Schwartz, M.W. (2007) A framework for debate of assisted migration in an era of climate change. Conserv. Biol., 21, 297-302.

Morgan, M.G. \& Dowlatabadi, H. (1996) Learning from integrated assessment of climate change. Climatic Change, 34, 337-368.

Morgan, M.G. \& Henrion, M. (1990) Uncertainty: a guide to dealing with uncertainty in quantitative risk and policy analysis. Cambridge University Press, Cambridge, UK.

Opdam, P. \& Wascher, D. (2004) Climate change meets habitat fragmentation: linking landscape and biogeographical scale levels in research and conservation. Biol. Conserv., 117, 285-297.

Parker, K.A. (2008) Translocations: providing outcomes for wildlife, resource managers, scientists, and the human community. Restor. Ecol., 16, 204-209.

Peterson, G. (2000) Political ecology and ecological resilience: an integration of human and ecological dynamics. Ecol. Econ., 35, 323-336.

Polasky, S., Carpenter, S.R., Folke, C. \& Keeler, B. (2011) Decision-making under great uncertainty: environmental management in an era of global change. Trends Ecol. Evol., 26, 398-404.

Raiffa, H., Richardson, J. \& Metcalfe, D. (2007) Negotiation analysis: the science and art of collaborative decision making. Belknapp Press, Cambridge, USA.

Regan, H.M., Colyvan, M. \& Burgman, M.A. (2002) A taxonomy and treatment of uncertainty for 
ecology and conservation biology. Ecol. Appl., 12, 618-628.

Ricciardi, A. \& Simberloff, D. (2009) Assisted colonization is not a viable conservation strategy. Trends Ecol. Evol., 24, 248-253.

Richardson, D.M., Hellmann, J.J., McLachlan, J.S. et al. (2009) Multidimensional evaluation of managed relocation. Proc. Natl. Acad. Sci., 106, 9721-9724.

Roy, B. (1999) Decision-aiding today: what should we expect? Pages 1/1-1/35 in T. Gal, T.J. Stewart, T. Hanne, editors. Multicriteria decision-making: advances in MCDM models, algorithms, theory and applications. Kluwer, Boston, MA.

Sala O.E., Chapin, F.S., Armesto, J.J. et al. (2000) Global biodiversity scenarios for the year 2100. Science, 287, 1770-1774.

Swart, R., Bernstein, L., Ha-Duong, M. \& Petersen, A. (2009) Agreeing to disagree: uncertainty management in assessing climate change, impacts and responses by the IPCC. Climatic Change, 92, 1-29.

Travis, J.M.J. (2003) Climate change and habitat destruction: a deadly anthropogenic cocktail. Proc. R. Soc. Lond. B Biol. Sci., 270, 467-473.
Walker, W.E., Harremoës, P., Rotmans, J. et al. (2003) Defining uncertainty: a conceptual basis for uncertainty management in model-based decision support. Integr. Assess., 4, 5-17.

Walley, P. \& de Cooman, G. (2001) A behavioral model for linguistic uncertainty. Inf. Sci., 134, 1-37.

Walters, C. \& Maguire, J.-J. (1996) Lessons for stock assessment from the northern cod collapse. Rev. Fish Biol. Fish., 6, 125-137.

Van Houtan, K.S. (2010) Conservation, biology, and religion. Pages 270-272 in N.S. Sodhi, P.R. Ehrlich, editors. Conservation biology for all. Oxford University Press, New York, USA.

Wintle, B.A., Bekessy, S.A., Keith, D.A. et al. (2011) Ecological-economic optimization of biodiversity conservation under climate change. Nat. Climate Change, $\mathbf{1}$, 355-359.

Vitt, P., Havens, K. \& Hoegh-Guldberg, O. (2009) Assisted migration: part of an integrated conservation strategy. Trends Ecol. Evol., 24, 473-474.

Vitt, P., Havens, K., Kramer, A.T., Sollenberger, D. \& Yates, E. (2010) Assisted migration of plants: changes in latitudes, changes in attitudes. Biol. Conserv., 143, 18-27. 